Revue Revue de l'histoire des religions

de Ihistoire des religions

$4 \mid 2005$

Lieux de culte, lieux saints dans le judaïsme, le christianisme et l'islam

\title{
Les lieux de culte chrétiens et le sacré dans
} l'Antiquité tardive

Places of Christian worship and their sacralization in Late Antiquity

\section{Claire Sotinel}

\section{CpenEdition}

\section{Journals}

Édition électronique

URL : https://journals.openedition.org/rhr/4473

DOI : $10.4000 /$ rhr. 4473

ISSN : 2105-2573

Éditeur

Armand Colin

Édition imprimée

Date de publication : 1 octobre 2005

Pagination : 411-434

ISBN : 2200-92087-3

ISSN : 0035-1423

Référence électronique

Claire Sotinel, «Les lieux de culte chrétiens et le sacré dans l'Antiquité tardive », Revue de l'histoire des religions [En ligne], 4 | 2005, mis en ligne le 14 janvier 2010, consulté le 21 septembre 2021. URL : http://journals.openedition.org/rhr/4473 ; DOI : https://doi.org/10.4000/rhr.4473 


\section{Les lieux de culte chrétiens et le sacré dans l'Antiquité tardive}

L'auteur examine dans quelle mesure les lieux de culte chrétiens, conçus d'abord surtout comme des maisons de prières, sont devenus des lieux sacrés à la fin de l'Antiquité tardive. La recherche récente sur les lieux saints de Palestine a montré comment les lieux de mémoire du christianisme ont été investis d'une valeur sacrée. Le développement du culte des reliques a permis de dissocier la sacralité d'une localisation géographique spécifique. Bien que l'opposition exprimée par certains théologiens n'ait pas interrompu cette évolution, la sacralité des lieux de culte n'est pas encore une réalité acquise dans les Églises d'Occident à la fin de l'Antiquité tardive.

\section{Places of Christian worship and their sacralization in Late Antiquity}

The author examines how the places of Christian worship, first conceived as houses of prayer, increasingly came to be considered as sacred places at the end of Late Antiquity. Recent research on holy places in Palestine has shown how sites of Christian memory have been sacralized. The development of the cult of relics led to a dissociation between sacrality and geographical location, allowing for the transformation of any place of worship into a holy place. The opposition of some Christian theologians did not stop this evolution, but it was not achieved by the end of Late Antiquity. 
Si de nombreuses questions demeurent sans réponse à propos des premiers lieux du culte chrétien, on connaît bien dorénavant les étapes qui ont abouti à la conception d'édifices spécialisés, ceux que nous appelons génériquement les églises ${ }^{1}$. Pendant la plus grande partie des trois premiers siècles du christianisme, les chrétiens ne bâtissent pas d'édifices spécifiques, se contentant d'utiliser des maisons d'habitation ${ }^{2}$ en adaptant au besoin au culte tout où partie de bâtiments existants ${ }^{3}$. Le seul cas d'église pré-constantinienne connu par l'archéologie est la «maison des chrétiens » de Doura Europos, dont l'utilisation n'a entraîné de modifications que de l'architecture intérieure et de la décoration, sans rendre le bâtiment remarquable de l'extérieur ${ }^{4}$. Rien ne permet de savoir si certains des «temples », des « églises », des « maisons de prières » confisqués ou détruits par les persécuteurs du III et du début du IV ${ }^{\mathrm{e}}$ siècle avaient été construits spécifiquement pour le culte chrétien. Certains étaient assurément visibles, comme l'église de Nicomédie qui, selon Lactance, «était située sur un terrain élevé, en vue du Palais », et que Dioclétien regarda brûler le jour où il déclencha la grande persécution ${ }^{5}$. Cela ne veut pas dire qu'ils étaient intrinsèquement reconnaissables, encore moins qu'ils avaient été construits pour être des églises. Dans un cas peut-être unique, où nous savons que des chrétiens ont construit des « bâtiments pour servir d'église », la nécessité faisait loi puisque les confesseurs déportés aux mines de cuivre de Phaino n'ont bien sûr pas la ressource d'utiliser leur propre demeure. Le terme neutre par lequel Eusèbe désigne cette

1. Pour une synthèse récente : Noël Duval, «Problématique d'une architecture chrétienne au IV e siècle », REAug 35 (1989), p.308-313, et id., s.v. « Édifices de culte », in Angelo di Berardino dir., Dictionnaire encyclopédique du christianisme ancien, Paris, 1990, p.752-771.

2. $\mathrm{Au} \mathrm{II}^{\mathrm{e}}$ siècle, à Rome, Justin affirme au préfet qui l'interroge qu'il prêchait aux fidèles qui venaient le visiter dans sa demeure - un appartement dans un insula - et qu'il ne connaît pas d'autres rencontres que celles tenues chez lui : Justin martyr, Actes, ch. 2.

3. Aldo Nestori, «Riflessioni sul luogo di culto cristiano precostantiniano », Rivista di Archeologia Cristiana 75, 1-2 (1999), p. 695-709.

4. Carl H. Kraeling, The Excavations at Dura Europos, Final Report VIII, Part II. The Christian Building, New Haven, 1967.

5. Lactance, De la mort des persécuteurs, 12, 5. 
construction, des bâtiments (oikoi), ne permet évidemment aucune conjecture architecturale ${ }^{6}$.

Il faut attendre la victoire de Constantin sur Maxence en 312, la légalisation du christianisme et les libéralités impériales pour voir apparaître les premiers lieux de culte par destination. D'emblée, ces monuments présentent une certaine variété ; lorsqu'il évoque génériquement les constructions chrétiennes de l'empereur à Constantinople, Eusèbe écrit : «Pour honorer avec une distinction exceptionnelle la cité qui porte son nom, il l'embellit avec de très nombreux lieux de culte, de très vastes martyriums et de splendides édifices, certains dressés en dehors de la cité, d'autres à l'intérieur » ${ }^{7}$. De fait, dès avant la paix de l'Église cohabitent des lieux destinés à la réunion de la communauté pour la célébration du culte et l'enseignement (ce sont les «lieux de prières » mentionnés par Eusèbe lorsqu'il évoque les reconstructions d'églises après la persécution ${ }^{8}$ ), des lieux de commémoration, construits sur les tombes des martyrs, et le plus souvent des ensembles complexes associant lieux de culte, bâtiments de service ou de résidence de l'évêque, etc.

Nous avons conservé des vestiges matériels de plusieurs édifices construits dans la première moitié du IV siècle : la basilique double d'Aquilée, celle de Trèves, les basiliques constantiniennes de Rome, l'église d'Ostie, les lieux saints de Palestine. Les témoignages littéraires et la documentation épigraphique attestent de nombreuses constructions antérieures à la période théodosienne en Afrique, Italie, en Orient, même s'il est le plus souvent difficile de dater précisément les monuments conservés ${ }^{9}$. À partir du $\mathrm{V}^{\mathrm{e}}$ siècle, ces monuments se multiplient dans toutes les provinces de l'empire, au point de modifier la topographie des cités. Avec la translation des reliques, introduite en Occident à la fin du IV siècle par Ambroise

6. Eusèbe de Césarée, De martyribus Palestinae 13, 1.

7. Eusèbe, VC III, 47.

8. Eusèbe de Césarée, Histoire Ecclésiastique, 10, 4.

9. John Bryan Ward Perkins, « Constantine and the origin of the Christian basilica ", Papers of the Bristish School in Rome 22 (1954) = Studies in Roman and Early Christian Architecture, Londres, 1994, p. 455-456 ; Noël Duval, "Problématiques d'une architecture chrétienne au IV siècle », Revue des Études Augustiniennes 35 (1989), p. 308-313. 
de Milan, la distinction entre églises et "sanctuaires », entendus comme « un édifice de culte dans lequel sont conservées les reliques sacrées, d'importance particulière, liées généralement à des événements miraculeux... ${ }^{10}$ tend à s'atténuer : par la déposition des reliques, toute église devient sanctuaire, même si toutes ne sont pas l'objet de pèlerinages « qui transcendent un lieu local ou régional » comme le dit la fin de la définition citée plus haut. La déposition des reliques dans les églises est de règle au $\mathrm{VI}^{\mathrm{e}}$ siècle, même si elle n'est pas une condition nécessaire à la dédicace ${ }^{11}$.

Les édifices de culte ne font guère, tout au long de cette période, l'objet d'un discours structuré et leur transformation - évidente au regard de l'observation des monuments eux-mêmes - n'a pas fait l'objet d'une analyse ou d'une réflexion contemporaine. Ni la « révolution édilitaire » que représente leur apparition et leur multiplication, ni même la concurrence qui s'établit au ve siècle entre monumentalité chrétienne et monumentalité civique - y compris celle des temples de la vieille religion -, ni l'élaboration d'une liturgie propre à la dédicace des églises n'ont inspiré les auteurs de l'Antiquité tardive, chrétiens ou non. Libanius a écrit un discours « sur les temples »; il n'existe pas de discours «sur les églises». Cependant, la documentation ne manque pas pour explorer le sujet. Les panégyriques prononcés à l'occasion de dédicaces d'églises, à commencer par celle de la basilique de Tyr en 315, les inscriptions qui ornent les monuments, des notations éparses dans des sermons au peuple, quelques règles énoncées dans les conciles, l'observation même des monuments conservés fournissent ample matière à la réflexion. Celle que je poursuis ici porte sur la façon dont la nature

10. Moreno Morani, «Sull'espressione linguistica dell'idea di santuario nelle civiltà classiche » in Marta Sordi dir., Santuari e politica nel mondo antico, Milan, 1983, p. 9, cité par Letizia Ermini Pani, "Il cristianesimo nell'antichità e nell'alto medioevo », in André Vauchez dir., Lieux sacrés, lieux de culte, sanctuaires. Approches terminologiques, méthodologiques, historiques et monographiques (Collection de l'École française 273), Rome, 2000, p. 108. La définition de Morani se poursuit par « ... et qui est devenu un but de pèlerinage en en général de dévotion qui transcende un lieu local ou régional ».

11. Les textes normatifs, comme nous le verrons plus loin, continuent d'envisager le cas d'églises dépourvues de reliques. 
du lieu de culte est perçue dans ses relations avec la communauté chrétienne et avec la pratique religieuse, sur la façon dont cette perception change avec le temps jusqu'à la « fin du christianisme ancien », sur les mécanismes de ces changements et sur les influences qui les ont inspirés. Sous-jacente à cette réflexion est l'interrogation suivante : l'église chrétienne devient-elle un lieu sacré au cours de l'Antiquité tardive, tend-elle à le devenir et, si oui, quand ?

\section{L'HISTOIRE PRAGMATIQUE DE L'APPARITION DES LIEUX DE CULTE}

Le lieu de culte chrétien est avant tout un lieu de rassemblement de la communauté, un lieu de prière et d'enseignement ${ }^{12}$. Le pragmatisme qui avait inspiré les aménagements cultuels chrétiens avant 312 domine aussi les premières constructions de la paix de l'Église. Beaucoup des nouvelles églises sont des reconstructions de bâtiments détruits pendant la persécution, comme le précise Eusèbe dans la Vita Constantini ${ }^{13}$. Déjà dans la Didascalie des apôtres ${ }^{14}$ ou les Constitutions apostoliques ${ }^{15}$ l'intérêt porté à la topographie du lieu de culte ne concernait que l'organisation des fidèles pendant la liturgie. Les réalisations matérielles confirment cette fonction première du lieu de culte.

Les modèles architecturaux choisis pour les plus anciennes églises reflètent principalement ce caractère. Il n'est pas question de suivre le modèle des temples païens, où le bâtiment n'abrite que les statues divines et les prêtres initiés. Il n'est pas question non plus de s'inspirer du modèle architectural du temple de Jérusalem, dont la destruction n'a fait que sanctionner matériellement sa disqualification

12. Antonio Quacquarelli, «I luoghi di culto e il linguaggio simbolico nei primi due secoli cristiani », RAC 42 (1966 [1968]), p. 237-266.

13. Eusèbe, $V C$, III, 1 : «Ils (les persécuteurs) détruisirent complètement les lieux de culte, en les démolissant du plafond au sol ; il (Constantin) décréta que ceux qui existaient devaient être agrandis, et de nouveaux érigés sur une grande échelle aux frais du trésor impérial ».

14. Didascalia apostolorum, 12.

15. Constitutions apostoliques, II, 57, 2. 
comme «maison de Dieu » ${ }^{16}$. Comme on le sait, les chrétiens se sont principalement inspirés de modèles de l'architecture civile ${ }^{17}$. Le Latran et St-Pierre à Rome, l'église constantinienne de Trèves et celle reconstruite à Tyr sont des basiliques dont les nefs s'ouvrent sur le petit côté, tandis que le côté opposé se termine en abside ; ce modèle basilical est au $\mathrm{IV}^{\mathrm{e}}$ siècle le plus répandu, au point que le mot basilica est souvent utilisé pour désigner une église ${ }^{18}$. À Aquilée, la plus ancienne église connue est composée de deux salles rectangulaires réunies par une troisième, plus petite ; ce plan d'église-halle connaît dans toute la région un succès constant jusqu'au ve siècle. L'église impériale d'Antioche a, elle, un plan centré, mais aussi est-elle conçue comme un mémorial, non comme une église ${ }^{19}$. Le caractère fonctionnel de ces bâtiments n'interdit pas qu'on cherche à leur conférer une valeur symbolique. Celle-ci s'exprime peut-être déjà dans le décor, mais le langage iconographique chrétien est encore balbutiant ${ }^{20}$; elle est surtout formulée dans les discours panégyriques, qui donnent des églises de la paix constantinienne une image qui peut être trompeuse si on les prend au pied de la lettre.

Ainsi, dans son célèbre discours pour la dédicace de la basilique de Tyr, Eusèbe multiplie les références au Temple de Jérusalem.

16. Épître de Barnabé, ch. 16 ; pour la polémique anti-juive au IV siècle : Rainer Kampling, «Die Darstellung der Juden und des Judentums in den Predigten des Zeno von Verona », Kairos 26 (1984), p. 10-24.

17. Noël Duval, «problématique d'une architecture religieuse du Ive siècle », REAug 35 (1989), p. 308-313, repris par Michel Yves Perrin, op. cit., note 35, p.589-591.

18. Christine Mohrman, «Les dénominations de l'église en tant qu'édifice en grec et en latin au cours des premiers siècles chrétiens ", in Études sur le latin des chrétiens, IV, Rome, 1977, p.211-230.

19. Eusèbe, $V C 3,50$ : « là, comme pour couronner les provinces, il consacra une église unique en taille et en beauté, entourant tout le temple d'une vaste enceinte extérieure, et érigeant la maison du culte à une énorme hauteur. Elle fut construite de taille octogonale, avec un anneau de fenêtres construites juste au niveau du sol et au premier étage, et il l'entoura avec de riches décors abondant en or, en bronze et toutes sortes de matériaux précieux. »

20. Je ne rentrerai pas ici dans la question un peu dépassée du prétendu parti pris aniconique du christianisme ancien. 
L'église est nommée « saint temple de Dieu », «maison (de Dieu) sur la terre ${ }^{21}$ et l'évêque qui a pourvu à sa construction est un « nouveau Béséléel, constructeur d'une tente divine, ou encore Salomon, roi d'une Jérusalem nouvelle, de beaucoup supérieure à l'ancienne, ou encore Zorobabel, qui apporte une gloire beaucoup plus grande que la première au temple de Dieu ${ }^{22}$. Mais cette comparaison - destinée à un avenir durable, puisqu'on la retrouve dans la liturgie de la dédicace des églises jusqu'au concile de Vatican II - ne doit pas faire illusion. Eusèbe met l'accent sur la sainteté de la communauté qui forme l'Église, dont le bâtiment, dans sa splendeur, n'est qu'une image. Le discours d'Eusèbe est à la fois un panégyrique impérial - Constantin a financé la reconstruction de la basilique de Tyr - et un discours pastoral - il est prononcé devant l'assemblée du peuple et plusieurs évêques. Il magnifie l'entreprise du prince, image de Dieu sur la terre, mais il insiste surtout sur une image déjà classique à l'époque dans le discours patristique, celui du «temple vivant», le «temple animé que vous êtes tous », la «maison faite de pierres vivantes et bien fixées, qui est fortement et solidement établie "sur le fondement des apôtres et des prophètes, Jésus-Christ lui-même étant la pierre angulaire" qu'ont rejetée non seulement les artisans de cette maison ancienne qui n'est plus, mais encore ceux de la construction faite par la plupart des hommes et qui subsiste jusqu'à présent, architectes des œuvres mauvaises $»^{23}$.

L'insistance sur le caractère métaphorique du bâtiment église, et sur son absence de valeur intrinsèque, s'est heurtée, au moins depuis la fin du IV siècle, à une certaine évolution de la sensibilité chrétienne. Cette dernière, sur laquelle nous reviendrons, tend à voir dans l'église un lieu religieusement privilégié, où la présence divine est plus forte qu'ailleurs. Du coup, le rappel du caractère neutre des lieux prend parfois l'allure d'une mise en garde. À la fin du IV siècle, l'évêque Zénon souligne avec satisfaction l'infériorité

21. Eusèbe de Césarée, $H E X, 4,2$.

22. Eusèbe de Césarée, $H E X, 4,3$.

23. Eusèbe de Césarée, $H E, X, 4,21$. 
esthétique de la nouvelle église de la communauté de Vérone par rapport à la splendeur des temples païens, pourtant déjà largement délaissés, pour mettre en valeur la vigueur et la beauté de la communauté chrétienne ${ }^{24}$; Augustin répète avec force cette distinction entre les pierres de constructions et les pierres spirituelles ${ }^{25}$, tandis que son attitude pastorale face à l'inhumation privilégiée dans les églises confirme sa réticence devant toute tentative de sacraliser le lieu ecclésial ${ }^{26}$. De tels discours vont assurément à l'encontre de la sensibilité la plus communément répandue. Pourtant, contrairement à ce que pourrait faire croire une lecture trop rapide des sources, ce refus tenace de la sacralisation du sanctuaire ne disparaît pas rapidement. Il est peut-être imprudent de considérer que le triomphe du mot ecclesia contre le mot dominicum en témoigne, mais le fait est que le vocabulaire du temple est resté confiné à la poésie, il est vrai aussi à la poésie épigraphique ; les mots aedes ou templa présents sous les yeux des fidèles peuvent avoir eu un certain impact $^{27}$. Mais on le voit plus sûrement encore dans le pragmatisme qui domine les règlements ecclésiastiques concernant les églises : construire une nouvelle église est affaire de nécessité, cela exige des conditions matérielles, en particulier la constitution de rentes pour son entretien, mais il n'y a aucun obstacle à l'abandon d'une église trop gravement endommagée par un incendie. Ce pragmatisme, souvent exprimé par Grégoire le Grand dans sa correspondance, est conservé dans le Liber Diurnus ${ }^{28}$ : le souci romain est d'assurer un contrôle des lieux de culte (donc, l'autorisation de l'évêque est

24. Zénon, Tractatus II, 6 (I, 4); Claire Sotinel, «Locus orationis ou domus Dei ? Le témoignage de Zénon de Vérone sur l'évolution des églises (tractatus II, 6) », Studia Patristica 29 (1997), p.141-147.

25. En particulier dans les sermons 252 et 336, qui sont passés dans la liturgie de dédicace des églises.

26. Éric Rebillard, «Violations de sépulture et impiété dans l'Antiquité tardive ", in L. Mary, Michel Sot éds, Impies et païens entre Antiquité et Moyen Âge, Paris, 2002, p. 74-76.

27. Claire Sotinel, «Locus orationis ou domus Dei ?», op. cit. note 24, p. $145-147$.

28. Liber Diurnus, formules X et XI (pour la fondation d'oratoires privés) et surtout XVIII (pour les basiliques). 
indispensable ${ }^{29}$, de garantir leur pérennité (il faut donc que le fondateur fournisse les fonds nécessaires pour la basilique et son éclairage). Certes, il faut aussi qu'aucun corps n'y soit enterré, mais l'interdit est formulé au même titre que la nécessité de veiller à ce que le terrain soit libre de toute contrainte fiscale. On ne trouve en revanche rien qui puisse être assimilé à la définition d'un espace sacré, contrairement à ce qu'exprime le rituel de dédicace des églises médiévales.

La constitution progressive d'un rite de dédicace et la pratique de dépôt des reliques dans les autels est-elle en contradiction avec cette conception pragmatique du lieu de culte et représente-t-elle une concession à une tendance vers sa sacralisation ? La réponse à cette question ne peut pas être simple et, pour l'approcher, il faut faire le détour par l'analyse des lieux saints du christianisme.

\section{LIEUX DE CULTE ET LIEUX SAINTS}

Qu'il y ait des lieux saints pour le christianisme ne va pas de soi, puisqu'il existe tout un enseignement, depuis les évangiles, pour contester la possibilité même qu'il existe des lieux privilégiés de la relation à Dieu ${ }^{30}$. Une certaine tradition chrétienne enseigne un « désenchantement du monde » qui aboutit à une totale intériorisation et humanisation du fait religieux. Cependant, dès avant le IV siècle, certains lieux de mémoire acquièrent une valeur particulière. Les uns sont ce qui deviendra les « lieux saints de Palestine », les lieux marqués par la présence terrestre de Jésus ; les autres sont les lieux

29. L'exigence est déjà exprimée dans une lettre de Gélase I aux évêques de Lucanie (A. Thiel, Epistolae Romanorum pontifium, Braunsberg, 1867 (rééd. 1974), p. 364) : basilicas nouiter institutas, non petitis ex more praeceptionibus, dedicare nemo audeat ; évidemment, à cette époque, la règle ne concerne que les évêques d'Italie suburbicaire. Il n'est pas certain que tous les métropolitains la pratiquent ailleurs et il est fort possible que dans la plupart des endroits, seul le contrôle épiscopal soit nécessaire, comme l'affirme d'ailleurs le canon 37 du concile d'Orléans de 437.

30. Comme l'écrit Robert A. Markus, The End of Ancient Christianity, Cambridge, 1993, p. 139, avant le Iv e siècle, "Christians inhabited a spatial universe spiritually largely undifferentiated ». 
associés aux martyrs, en général leur tombe, plus rarement - et souvent plus tardivement - le lieu de leur supplice.

La littérature sur les lieux saints de Palestine s'est considérablement renouvelée ces dernières années. Comme l'a bien montré P. W. L. Walker, toute une tradition, dignement représentée par Eusèbe de Césarée, voit dans les «lieux de Jésus » de simples mémoriaux : "Assurément, il n'y avait plus de lieu comparable au Temple de l'Ancien Testament, qui avait été "saint" au sens de "spécial aux yeux de Dieu", un lieu spécial pour sa présence et sa rencontre avec son peuple ; de telles idées, attribuant un statut divin inhérent ou ontologique de sainteté à un lieu spécifique, avaient été accomplies et déplacées dans le Christ. Pourtant, il était peut-être permis de parler encore de "lieux sacrés" en un sens plus faible, comme un moyen utile d'exprimer la signification historique et religieuse de lieux particuliers. » 31

Une telle approche se retrouve encore plusieurs décennies plus tard chez un Jérôme qui vante avant tout le caractère édifiant de la Palestine, tant sur le plan moral qu'intellectuel ${ }^{32}$. Mais, parallèlement à cette attitude, la construction d'édifices de culte sur ces lieux saints de Palestine par Constantin a introduit une nouvelle approche de la sainteté du lieu. À la mémoire s'ajoute une qualité spécifique au lieu «contaminé » par le saint qui l'a fréquenté. Selon Walker, pour Cyrille de Jérusalem, ces lieux avaient gagné une qualité inhérente de sainteté à travers cette association divine à l'époque de l'incarnation. «Pour ceux qui ont la foi, elles ont une

31. Peter W. L. Walker, Holy City, Holy Places? Christian attitudes to Jerusalem and the holy land in the fourth century, Oxford, 1999, p. 41-42.

32. Jérôme, dans l'oraison funèbre qu'il compose pour Paula (=Ep. 108), décrit son pèlerinage aux loca sancta de Palestine comme un récapitulatif de l'histoire biblique. Dans l'introduction aux Lib. Paralip., le même auteur écrit : " De la même façon que ceux qui ont vu Athènes comprennent mieux l'histoire des Grecs, et que ceux qui ont fait voile depuis Troie par Leucate, et de l'Acrorénauna jusqu'en Sicile, et de là jusqu'à l'embouchure du Tibre comprennent mieux le troisième livre de Virgile, ainsi, ceux qui ont contemplé la Judée de leurs propres yeux et connaissent les sites des anciennes cités et connaissent le nom des lieux, que ce soit le même ou qu'il soit changé, regarderont l'écriture avec plus de lucidité ». Cité par Joan E. Taylor, Christians and Holy Places : The Myth of Jewish-Christian Origins, Oxford, 1993 p. 308. 
certaine puissance spirituelle, ou même une signification "sacramentelle", en tant que lieux que Dieu peut utiliser pour transmettre un sens spécial de sa présence »; «(ces "lieux saints") étaient en soi des médiums appropriés de la foi, des endroits où le divin avait touché l'humain, le physique, des endroits où à travers les moyens matériels du toucher, de la vue et de l'action liturgique, les êtres humains pouvaient maintenant redevenir proches du divin ${ }^{33}$. Ce caractère inhérent de la sainteté et du lieu est affirmé par Constantin à propos du Golgotha, comme le fait remarquer le même auteur ; lorsqu'il écrit à l'évêque Macarios, l'empereur parle d'un lieu saint aux yeux de Dieu depuis le commencement, et devenu plus saint encore $^{34}$. La sainteté de ces lieux est source de miracles, elle inspire des pèlerinages. Les lieux de culte construits dépendent de la sainteté des emplacements où ils sont construits; ils s'inscrivent ainsi eux aussi dans la géographie du sacré.

Un phénomène voisin se produit avec d'autres lieux de mémoire, les tombes des maryrs. Il n'y a pas de lien généalogique entre ces derniers et les lieux saints de Palestine puisque certaines tombes ont été vénérées dès le $\mathrm{III}^{\mathrm{e}}$ siècle, avant que les chrétiens aient pu être en mesure de s'approprier les lieux saints de Palestine. Mais le processus est assez semblable, en ce que c'est la mémoire, le souvenir d'un événement particulier qui insuffle au lieu sa sainteté. Le caractère funéraire de ces sanctuaires est d'autant plus marqué que c'est la célébration anniversaire de la déposition qui rassemble les fidèles, souvent sous l'autorité de l'évêque ; la construction de lieux de culte sur de tels lieux relève d'un double souci de monumentalisation du mémorial et de rassemblement d'une communauté qui peut être nombreuse au moment de la fête du saint. Dans ce registre, maîtrise de l'espace et maîtrise du temps sont étroitement liés dans le développement du férial et de la liturgie stationnaire ${ }^{35}$.

33. Joan E. Taylor, Christians and the Holy Places, p. 37-38.

34. Eusèbe, $V C$ 3, 30, 4, cité par J. E. Taylor, op. cit., p. 48.

35. Voir le titre significatif de la contribution de Michel Yves Perrin, «Le nouveau style missionnaire. La conquête de l'espace et du temps », in Jean-Marie Mayeur et alii (dir.), Histoire du christianisme. 2. Naissance d'une chrétienté (250-430), Paris, 1995, p.585-621, synthétisant, entre autres, l'enseignement de Ch. Pietri. 
Au-delà du rôle pastoral de cette conquête du temps, R. Markus a bien montré comment le développement d'une géographie du sacré avait répondu pour les chrétiens des $\mathrm{IV}^{\mathrm{e}}$ et $\mathrm{V}^{\mathrm{e}}$ siècles au besoin pressant de combler ce qui pourrait les séparer de l'Église sainte du temps des martyrs et réalisant une projection géographique de la sacralisation du temps ${ }^{36}$. Avec les saints d'un nouveau type que sont les ascètes ou les saints évêques, ce n'est plus le lieu de la mort seul qui devient chargé de sainteté, mais les lieux de la vie du saint : la construction du complexe de Saint-Siméon illustre le lien devenu ordinaire entre mémorial de sainteté et édifications des lieux de culte. Mais en Gaule au vi ${ }^{\mathrm{e}}$ siècle, Grégoire de Tours montre que ce lien n'est pas nécessaire. Il emploie le nom de loca sanctorum ou de loca sancta pour désigner tous les lieux qu'un saint a de son vivant sacralisés par sa présence et qui sont par là même porteurs de vertu miraculeuse : à Tours, le monastère de Marmoutier, mais aussi la terre foulée par le saint, l'eau qu'il a bue ${ }^{37}$.

Mais, à partir de la fin du IV siècle, le rapport entre sanctuaires et lieux de culte change avec la pratique de la déposition des reliques dans les églises. Le cas le plus éclatant, le mieux connu et le premier en Occident, est celui de la déposition des reliques des saints Gervais et Protais à Milan. Le rapport entre lieu saint et lieu de culte est en effet inversé : il ne s'agit plus de construire une église sur un lieu saint, mais de faire de l'église un lieu saint en y introduisant un élément sanctifiant, pour ne pas dire sacralisant. Les reliques sont d'ailleurs souvent appelées sacraria, en particulier dans les textes normatifs relatifs à la consécration des églises.

La pratique de la déposition des reliques dans les églises a des conséquences variées sur la perception des lieux de culte et sur leur réalité matérielle. Elle fait de toute église un sanctuaire, répondant peut-être ainsi à une demande diffuse dans certains milieux chrétiens

36. Robert A. Markus, «How on earth could places become holy?», JECS 2 (1994), p. 257-271. Du même auteur, voir aussi, The End of Ancient Christianity, p.139-143.

37. Luce Pietri, " Grégoire de Tours et la géographie du sacré », in Nancy Gauthier et Henri Galinié éds, Grégoire de Tours et l'espace gaulois : actes du congrès international, Tours, 3-5 novembre 1994, Tours, 1997, p.111-114. 
dont la sensibilité ne se satisfait plus de lieux de culte dont la sainteté n'est que métaphorique. Certains évêques prennent d'ailleurs des précautions contre un enthousiasme trop grand pour la transformation des églises, par la vertu contaminante des reliques, en lieux saints. Ainsi, Chromace d'Aquilée, dans le sermon qu'il prononce pour la dédicace de la nouvelle cathédrale de Concordia, parle des reliques en une formule ambiguë. L'Église de Concordia se voit ornée « des reliques des saints, de la construction de la basilique et du ministère $\mathrm{du}$ grand prêtre ${ }^{38}$. Les reliques des saints à elles seules, pas plus que l'établissement d'un évêque, ne sont constitutifs de l'Église de Concordia. Certes Chromace prononce longuement l'éloge des saints dont les reliques sont vénérées à Concordia, mais après avoir pris la précaution de noter que leur foi et leur gloire brillent par toute la terre et que leurs vertus et leur grâce opèrent en tous lieux ${ }^{39}$ et il précise que les chrétiens ne doivent pas attendre « de la part des Apôtres une grâce moindre du fait que nous n'avons pas les reliques de chacun d'eux ${ }^{40}$.

Une telle prudence cependant, si elle continue à structurer le discours clérical sur la nature des lieux de culte, n'empêche pas l'église de se transformer dans sa réalité matérielle. L'introduction des sacraria (les reliques) entraîne des aménagements (reliquaires, cryptes), des gestes (onction de l'autel, procession de déposition des reliques) et des pratiques (pèlerinages, vénération des reliques) qui hiérarchisent l'espace intérieur de l'église ${ }^{41}$ et en modifient la

38. Chromace, Sermon 26, 1, SC 164, p.94 : Ornata est igitur Ecclesia Concordiensis et munere sanctorum, et basilica constructione, et summi sacerdotis officio.

39. Ibid., 2 : Dignum est enim nos aliquid de eorum meritis praedicare, quorum fides et gloria toto orbe refulget, quorum uirtus et gratia ubique operatur.

40. Ibid. : Sed nec in eo minorem apostolorum gratiam expectamus, quod non omnium reliquias habere uideamur. Traduction J. Lemarié, SC 164, p.95.

41. Les conciles d'Epaone (517), canon 26, d'Agde (506), canon 14 et de Séville (619) canon 7 réglementent l'onction de l'autel ; pour les pèlerinages, voir pour l'Occident Gisela Cantino Wataghin et Letizia Pani Ermini, «Santuari martiriali e centri di pellegrinaggio in Italia fra tarda antichità e altomedioevo », in Akten des XII. Internationalen Kongresses für christliche Archäologie, Bonn, 22-28 September 1991, Münster, 1996, p.123-151. 
perception. Elle abolit au moins en partie la distinction entre les églises funéraires et les autres : l'inhumation ad sanctos devient possible dans toutes les églises ${ }^{42}$.

Il n'est pas impossible qu'au moins une partie de cette évolution s'explique par une réaction à la politique anti-chrétienne de l'empereur Julien entre 360 et 363 . En affichant sa volonté de resacraliser des espaces qu'il considère profanés par les chrétiens (Antioche, Jérusalem), Julien a pu donner involontairement une plus grande légitimité au culte des reliques et à la reconnaissance de la sainteté des lieux où elles reposaient, de même qu'il ouvrait la porte à la concurrence entre églises et temples ${ }^{43}$.

Dans le même temps, le caractère de plus en plus systématique de l'introduction des reliques dans les églises peut être aussi compris comme une réaction à la séduction puissante qu'exercent les lieux saints, voire à la tentation de donner une valeur trop grande au sacré de lieux spécifiques. Si on peut le déplacer, sur une décision institutionnelle relevant de l'évêque (et, en Italie suburbicaire, du pape à partir de la fin du $\mathrm{V}^{\mathrm{e}}$ siècle ${ }^{44}$ ), les privilèges inhérents au lieu géographique sont moindres. Parallèlement, comme l'introduction des reliques relève d'une sanction institutionnelle et publique - au moins au sein de l'église - elle se rapproche des procédures romaines de sacralisation d'un espace : par une décision du peuple romain (ici chrétien), un espace reçoit une qualité particulière liée à son rapport

42. Je ne rentrerai pas ici dans la discussion sur cette vaste question : Yvette Duval et Jean-Charles Picard éds, L'inhumation privilégiée du IV au VIII ${ }^{e}$ s. en Occident. Actes du colloque tenu à Créteil les 16-18 mars 1984, Paris, 1986 ; Yvette Duval, Auprès des saints, corps et âme. L'inhumation "ad sanctos » dans la chrétienté d'Orient et d'Occident du III au VII siècle, Paris, 1988.

43. C'est ce que suggère Francesco Gandolfo, « Luoghi dei santi e luoghi dei Demoni », in Santi e demoni nell'alto medioevo occidentale (secoli V-XI) (36 ${ }^{\text {e }}$ SSCISAM), Spolète, 1989, p.883-916. Certains auteurs rapportent des profanations de tombes de martyrs (Jean-Baptiste en Palestine, Patrophile à Scythopolis : Chronicon Paschale, 285 ; Philostorge, 7, 4; Sozomène, 5, 5) et d'églises (une statue de Dionysios dans l'église d'Emèse : Chronicon Paschale, 285 ; Théohpanes 48, 12-13).

44. Gélase, Ep. citée note 29. 
avec le divin ${ }^{45}$. Ainsi, paradoxalement, alors que le développement des lieux saints en Palestine pourrait avoir renforcé l'influence du modèle vétéro-testamentaire du temple, l'introduction presque systématique des reliques dans les lieux de culte chrétiens les rapproche plutôt du modèle du temple romain.

\section{LIEUX DE CULTE ET PAYSAGE URBAIN}

Lieu de rassemblement de la communauté, lieu sanctifié par la prière qui s'y élève et par la célébration de l'eucharistie, souvent sacralisé par la contamination des reliques qui y sont déposées, les lieux de culte chrétiens sont aussi des lieux visibles qui expriment la place du christianisme dans les communautés humaines. La visibilité des lieux de culte est un enjeu important de leur développement. Avant le $\mathrm{IV}^{\mathrm{e}}$ siècle, la discrétion est une vertu inspirée par une dure nécessité. Après sa conversion, Constantin préside à l'érection d'une série d'églises "vastes et splendides » ${ }^{46}$ à Rome, Trèves, Constantinople, Tyr, Antioche et en Palestine. La plus ancienne, la basilique du Latran, concurrence les constructions publiques de Rome par ses dimensions $(98 \times 56 \mathrm{~m})$ et la richesse de ses matériaux de construction ; mais elle se situe à la périphérie de l'espace urbain qu'elle ne transforme pas, non plus que Saint-Pierre du Vatican, église suburbaine construite sur une zone funéraire. Les autres constructions romaines de période constantinienne sont encore plus discrètes : Sainte-Croix de Jérusalem, construite en ville, mais dans un espace privé, les basiliques cémétériales des catacombes de la via Appia (saint

45. Annie Dubourdieu, John Scheid, «Lieux de culte, lieux sacrés : les usages de la langue. L'Italie romaine », in André Vauchez éd., Lieux sacrés, lieux de culte, sanctuaires, op. cit. note 10, p. 60 : est sacer « tout ce qui est considéré comme la propriété des dieux », donc « ce qui a été dédié et consacré aux dieux ». Ce n'est pas une qualité divine, mais une qualité que les hommes y mettent. Ainsi, les dieux ne sont pas sacrés, et aucun objet ne peut être considéré comme divin. Le sacré est une qualité juridique que cet objet possède du fait d'une décision humaine.

46. Richard Krautheimer, Rome, portrait d'une ville, trad. Françoise Monfrin, Paris, 1999, (Princeton, 1980), p.53. 
Sébastien), Tiburtina (saint Laurent), Labicana (Marcellin et Pierre) ou Nomentana (Agnès) ${ }^{47}$. À Constantinople, la très vaste basilique plus tard dédiée à sainte Sophie est plus centrale dans le plan de la nouvelle capitale impériale, mais nous la connaissons très mal ${ }^{48}$ et la seule église constantinienne achevée sous son règne est celle de son mausolée, les Saints-Apôtres, un édifice qui est aussi un mémorial, comme l'église à plan centré d'Antioche, et qui est justement situé sur le point le plus élevé de la ville ${ }^{49}$. Dans le cas des constructions impériales, la monumentalité de l'église exprime la grandeur de l'empereur autant que celle de sa nouvelle religion. Mais pour bien des chrétiens, la monumentalité de l'église a pour vocation de refléter la puissance nouvelle de l'Église. Le discours d'Eusèbe pour la basilique de Tyr est à cet égard éloquent : si l'église est bien « seulement » une figure de la communauté chrétienne, sa splendeur doit refléter la sainteté de cette dernière.

Il serait cependant erroné de mesurer l'impact architectural des églises chrétiennes à l'aune des splendides réalisations impériales. Bien des églises du IV $V^{\mathrm{e}}$ siècle conservent quelque chose de la discrétion des «maisons d'église » antérieures à la paix constantinienne. Les églises titulaires de Rome « ne se distinguaient en rien des centaines d'insulae, des vieilles domus, des magasins ou des boutiques des quartiers populaires ; et du fait de leur rareté - seulement vingtcinq encore au $\mathrm{IV}^{\mathrm{e}}$ siècle -, elles passaient inaperçues au milieu des quarante-quatre mille insulae de la Rome contemporaine $»^{50}$. Le complexe épiscopal d'Aquilée, construit avec la bénédiction de Constantin sinon avec son appui matériel, se fond entièrement dans

47. Richard Krautheimer, Rome, portrait d'une ville, p. 54-66 ; voir aussi Louis Reekmans, «L'implantation monumentale chrétienne dans le paysage urbain de rome de 300 à 850 », in Actes du XIe Congrès international d'archéologie chrétienne. Lyon, Vienne, Grenoble, Genève et Aoste (21-28 septembre 1986), Rome-Cité du Vatican, 1989, p.861-915.

48. Richard Krautheimer, Tre capitali cristiane. Topografia e politica, Turin, 1987 (Berkeley-Los Angeles-Londres, 1983), p. 77-80.

49. Richard Krautheimer, Tre capitali cristiane, p.88-89.

50. Richard Krautheimer, Rome, portrait d'une ville, p.47. La remarque décrit la situation à la veille de l'arrivée au pouvoir de Constantin, mais elle vaut aussi pour la plupart des édifices du IV siècle. 
son environnement immédiat d'entrepôts ${ }^{51}$. Le sentiment que la discrétion monumentale des églises est une bonne chose est encore exprimé par Zénon de Vérone qui, à la fin du IV siècle, se réjouit de voir que la nouvelle église de sa cité ne cherche pas à rivaliser avec les splendeurs architecturales de la cité classique ${ }^{52}$. Il n'est pas impossible qu'une telle modestie reflète aussi le caractère minoritaire de la communauté des fidèles à Vérone, une situation encore fréquente à la fin du IV ${ }^{\mathrm{e}}$ siècle. En effet, une telle attitude est rare et tend à disparaître au profit d'une exaltation sans réserve de la splendeur expressive des monuments. Il suffit de citer, pour l'Occident, les poèmes de Prudence sur la basilique Saint-Paul à Rome ${ }^{53}$, de Paulin de Nole pour les basiliques en l'honneur de saint Félix ou, plus tard au vi ${ }^{\mathrm{e}}$ siècle, d'Ennode de Milan, pour voir comment peut disparaître toute réticence devant la magnificence architecturale. Mais on notera aussi que ces textes ressortissent à la poésie et que les deux types de discours peuvent coexister à la même époque. Se développe la tradition d'une ecphrasis des monuments chrétiens qui devient un genre littéraire en soi, destiné à un long avenir surtout dans la littérature byzantine. Jamais vraiment descriptifs, au grand dam des historiens de l'art, ces textes développent surtout une analyse symbolique de l'architecture des lieux de culte, analyse empruntée fondamentalement au modèle du Temple de Jérusalem ${ }^{54}$.

La valeur de signe que représentent les lieux de culte chrétiens ne se limite pas à exprimer, de façon globale et ostentatoire, la victoire de la religion du prince face à la société tout entière. Au fur et à mesure que le christianisme se diffuse dans chaque ville de l'Empire, les églises contribuent à modifier la topographie des cités. L'impact des constructions chrétiennes sur l'urbanisme a certainement été un des domaines les plus féconds des recherches

51. Paul-Albert Février, « Remarques sur le paysage d'une ville à la fin de l'Antiquité. L'exemple d'Aquilée », Antichità Altoadriatiche 19 (1981), p. 163212 ; C. Sotinel, Identité civique et christianisme. Aquilée du $\mathrm{III}^{e}$ au $\mathrm{VI}^{e}$ siècle, sous presse.

52. Claire Sotinel, «Locus orationis ou domus Dei ? », cité note 24.

53. Prudence, Peristephanon, 12, 49-54

54. Johnattan Z. Smith, To Take Place: Toward Theory in Ritual, Chicago, 1987, p. 47-115. 
sur les villes de l'Antiquité tardive ces dernières années, et il serait vain de prétendre ici résumer tous les acquis de ces travaux ${ }^{55}$. À partir de la fin du IV siècle, elle s'exprime en termes de compétition avec les autres édifices publics et, plus particulièrement, avec les temples païens. Certes, les communautés chrétiennes ne contribuent que ponctuellement à la destruction des temples et elles n'entreprennent que très tardivement - et selon un processus complexe de les réutiliser comme lieux de culte ${ }^{56}$, mais elles commencent plus tôt à occuper les espaces les plus centraux et les plus symboliques des cités antiques, en particulier le forum. La compétition pour la présence monumentale dans la cité ne se résume pas non plus à une rivalité entre christianisme et autres religions ; elle manifeste aussi les conflits entre différentes communautés chrétiennes, comme à Milan entre communautés nicéennes et ariennes sous l'épiscopat d'Ambroise ${ }^{57}$, en Afrique entre donatistes et catholiques ${ }^{58}$. Il est

55. Pour l'Occident, on notera les volumes publiés de la Topographie chrétienne des cités de la Gaule et le bilan de Pasquale Testini, Gisela Cantino Wataghin et Letizia Pani Ermini, La cattedrale in Italia, in Actes du XIe Congrès international d'archéologie cité note 49. Pour une bibliographie sommaire, voir Claire Sotinel, «Lieux de culte et sanctuaires dans le christianisme ancien. Enquête bibliographique », in Lieux sacrés, lieux de culte, sanctuaires, op. cit. note 10, p. 95-105.

56. Sur cette question très discutée, Laurence Foschia, «La réutilisation des sanctuaires païens par les chrétiens en Grèce continentale (IV ${ }^{\mathrm{e}}-\mathrm{VII}^{\mathrm{e}} \mathrm{s}$.), Revue des études grecques, 113 (2000), p.413-434 ; Béatrice Agnès Caseau, «POLEMEIN LITHOIS : la désacralisation des espaces et des objets religieux païens durant l'Antiquité tardive », in Michel Kaplan éd., Le sacré et son inscription dans l'espace à Byzance et en Occident : études comparées, Paris, 2001 (Série Byzatina Sorboniensia, 18), p. 61-123 ; Cl. Sotinel, « La disparition des lieux de culte païens en Occident. Enjeux et méthode », in Michel Narcy, Éric Rebillard éds, Hellénisme et christianisme, Villeneuve d'Ascq, 2004, p. 35-60.

57. Richard Krautheimer, Tre capitali cristiane, cité note 49, p. 107-148 ; Françoise Monfrin, « à propos de Milan chrétien. Siège épiscopal et topographie chrétienne IVe-VIe siècles », Cahiers Archéologiques 39 (1991), p. 7-46. L'invention des reliques de Gervais et Protais ressort aussi de cette concurrence entre les communautés.

58. La situation n'est pas nouvelle ; on se souvient que c'est pour le contrôle de « la maison de l'Église » d'Antioche, en 271/272, que les communautés chrétiennes de la ville avaient interpellé l'empereur Aurélien contre Paul de Samosate : Eusèbe, Histoire ecclésiastique, VII, 30, 18. 
significatif que les archéologues ne disposent d'aucun moyen pour reconnaître une église arienne ou donatiste d'un édifice nicéen ou catholique, mais il ne fait pas de doute que les contemporains, eux, faisaient la différence. La rivalité entre les différents groupes a d'ailleurs contribué aussi au changement de perception des espaces cultuels. Au Moyen Âge, le texte qui fait référence, pour fonder la pratique d'exorciser les églises avant la dédicace, est un texte de Grégoire le Grand qui rapporte comment, lors de la première messe célébrée dans l'église de Sainte-Agathe, autrefois église des ariens, le diable fut contraint de quitter l'édifice ${ }^{59}$. En recommandant à Mellitus d'exorciser les temples des Anglais avant de les utiliser pour le culte chrétien, le même pape a fourni ainsi à la liturgie de la dédicace un modèle durable ${ }^{60}$.

On pourrait dire que les lieux de culte ont des fonctions différentes selon qu'on les considère de l'intérieur, selon la qualité de l'espace qu'ils enclosent, ou de l'extérieur, comme les monuments qu'ils sont aussi. À ce dernier titre, on le voit, ils sont des enjeux de pouvoir et contribuent à donner forme visible à la place du christianisme dans la société. Mais cette visibilité n'est pas sans rapport avec la définition interne du lieu de culte : en plaçant les édifices chrétiens en concurrence matérielle avec d'autres lieux de culte, elle a conduit à des efforts de distinction (exorcisme, vite compris comme une « resacralisation ») et de définition, qui ont laissé une empreinte durable.

\section{VERS DES LIEUX DE CULTE SACRALISÉS}

Un dernier élément, à mon sens, a contribué à transformer les lieux de culte chrétiens de l'Antiquité ; il s'agit de leur statut juridique tel

59. Grégoire, Ep. 3, 19, ordonne au diacre Pierre d'envoyer des reliques de saint Séverin (du Norique) à Rome pour les déposer dans l'église qui doit être rendue au culte catholique ; dans les Dialogues, 3, 30, Grégoire rapporte la défaite diabolique ; ce dernier texte est cité par le canoniste Guillaume Durand pour formuler la première raison pour laquelle une église doit être dédiée selon les règles « ut dyabolus et eius potestas inde penitus expellatur»: Guillelmus Durantus, Rationale Divuinorum officiorum, I, 6, 5, CCcm 140, p.67.

60. Grégoire, Ep.11, 56. 
qu'il est reconnu par le prince. La question n'est pas simple et je veux l'aborder ici uniquement sous l'angle particulier de ce qu'on appelle le droit d'asile ${ }^{61}$. Un certain nombre de lois conservées dans le code théodosien définissent la protection que l'Église peut - ou ne doit pas - accorder à des fugitifs qui y chercheraient refuge. Le titre qui regroupe ces textes parle de ceux « qui ad ecclesias confugiunt $» 62$. La plupart de ces textes limitent l'intervention ecclésiastique en faveur de criminels condamnés, d'esclaves en fuite, de particuliers cherchant à se soustraire à leurs obligations ${ }^{63}$. Sans reprendre l' analyse d'un dossier complexe, je veux ici simplement souligner que le vocabulaire employé dans ces textes conserve une certaine ambiguïté. Avec la formule «ad ecclesiam confugere », ils ne disent pas si la protection découle de la présence dans un lieu particulier, ou si elle est accordée par les autorités ecclésiastiques. La loi du 27 juillet 398 semble indiquer que la question du lieu n'est pas primordiale, puisqu'elle évoque la possibilité que les personnes en butte aux poursuites puissent aussi bien chercher à se faire ordonner clerc que vouloir être défendues par un clerc ${ }^{64}$.

Cependant, les églises sont déjà considérées, de fait, comme un asile contre l'exercice de la force illégitime. Lors du sac de Rome par Alaric en 410, elles sont utilisées comme tel par la population et le roi barbare reconnait a posteriori cet asile qui, aux yeux des contemporains, a une valeur uniquement religieuse. Il désigne bien les églises comme des lieux spéciaux, où la protection de Dieu se manifeste d'une manière particulière.

Au moment de la compilation du Code Théodosien, en revanche, l'idée de l'asile conféré par les églises en vertu de leur caractère sacré défini par la loi est parfaitement assimilée. L'ensemble des

61. Anne Ducloux, "Ad ecclesiam confugere » : naissance du droit d'asile dans les églises : IVe-milieu du $v^{e}$ s., Paris, 1994.

62. CTh. 9, 45.

63. Anne Ducloux, «L'Église, l'asile et l'aide aux condamnés d'après la constitution du 27 juillet 398 », Revue historique de droit français et étranger, 69 (1991), p. 141-176. Il s'agit de CTh. 9, 45, 1 (392), 2 (397), 3 (398).

64. CTh. 9, 45, 3 : ...quilibet postremo publicis priuatisue rationibus inиolutus ad ecclesiam confugiens uel clericus ordinatus uel quocumque modo a clericis fuerit defensatus... 
textes que nous avons évoqués plus haut fait suite à la législation sur l'asile procuré par les statues impériales. Plus nettement encore, une série de lois de 431 définit positivement le droit d'asile en des termes nouveaux. Le premier de ces textes définit précisément les espaces qui protègent le fugitif de l'atteinte de la loi. Le vocabulaire qu'il emploie est entièrement emprunté aux lois romaines sur les espaces sacrés. Les églises sont « les temples du dieu très haut», les autels sont « sacro-saints » et porter la main sur les réfugiés est 《 sacrilège ${ }^{65}$. Ainsi, non seulement l'église est définie dans sa réalité matérielle de lieu de culte, mais elle est définie comme un espace sacré conformément au droit romain traditionnel tel qu'il s'appliquait aux temples de la religion civique. Les lois qui interdisent d'entrer en armes dans les églises où d'y exercer toute violence emploient le même vocabulaire ${ }^{66}$. Le commentaire du dernier de ces textes assimile les églises aux « lieux dédiés à Dieu » et aux « lieux saints », témoignant ainsi de l'aboutissement de l'évolution que nous avons essayé de retracer. Pour la loi impériale, les églises sont désormais assimilées aux temples en tant que lieux sacrés.

«Terribilis est locus iste », disait l'introït de la messe pour la fête de la dédicace, selon une tradition qui remonte peut-être à la consécration du Panthéon transformé en église par Boniface IV en $610^{67}$. Le rituel de la dédicace, tel qu'il est fixé au XIII ${ }^{\mathrm{e}}$ siècle, affirme explicitement le caractère spécial du lieu publiquement consacré, purifié de toute présence démoniaque, séparé du reste du monde et particulièrement empli de présence divine ${ }^{68}$. Mais en traçant une généalogie continue de la consécration du temple de Jérusalem aux rites chrétiens pratiqués à son époque, le canoniste Guillaume Durant se trompe, bien qu'il utilise pour sa démonstration des textes patristiques des $\mathrm{V}^{\mathrm{e}}$ et $\mathrm{VI}^{\mathrm{e}}$ siècles. Selon lui, les églises doivent être consacrées pour cinq raisons différentes : 1) que le diable en

65. CTh. 9, 45, 4a, adressée à Antiochus Chuzon (cf. PLRE 2, Antiochus 7 , p.103).

66. CTh. 9, 45, 4 1, 2 et 3 parlent des « temples du dieu très haut » et des « autels divins ». Datées du consulat d'Antiochus.

67. Iledfonse Schuster, Liber Sacramentorum 8, Turin, 1927.

68. Guillelmus Durantus, Rationale Divuinorum officiorum, I, VI, CCcm 140, p.63-84. 
soit chassé, 2) que ceux qui s'y réfugient y trouvent le salut, 3) que les prières y soient exaucées, 4) que les louanges y soient rendues à Dieu, 5) que les sacrements ecclésiastiques y soient administrés ${ }^{69}$. Nous avons vu comment, loin d'être une réalité inhérente au christianisme, ces caractéristiques des lieux de culte sont élaborées très progressivement et encore imparfaitement au cours de l'Antiquité tardive, sous les influences diverses du judaïsme, de la conception romaine du sacré et du développement interne du christianisme ancien. Ces influences ne suscitent pas une évolution linéaire, mais l'entrecroisement de discours et d'attitudes parfois contradictoires qui caractérisent tout au long de la période le christianisme ancien.

98, rue Jean-Pierre Timbaud

75011 Paris

\section{BIBLIOGRAPHIE}

Cantino Wataghin Gisela et Pani ERmini Letizia, « Santuari martiriali e centri di pellegrinaggio in Italia fra tarda antichità e altomedioevo », in Akten des XII. Internationalen Kongresses für christliche Archäologie, Bonn, 22-28 September 1991, Münster, 1996, p. 123-151.

CASEAU Béatrice Agnès, "POLEMEIN LIQOIS : la désacralisation des espaces et des objets religieux païens durant l'Antiquité tardive », in Michel Kaplan (éd.), Le sacré et son inscription dans l'espace à Byzance et en Occident : études comparées, Paris, 2001 (Série Byzantina Sorboniensia, 18), p. 61-123.

Dubourdieu Annie et ScheID John, «Lieux de culte, lieux sacrés : les usages de la langue. L'Italie romaine », in André Vauchez (éd.), Lieux sacrés, lieux de culte, sanctuaires, p. 59-80.

Ducloux Anne, "Ad ecclesiam confugere » : naissance du droit d'asile dans les églises (IVe-milieu du $v^{e}$ s.), Paris, 1994.

Ducloux Anne, «L'Église, l'asile et l'aide aux condamnés d'après la constitution du 27 juillet 398 », Revue historique de droit français et étranger, 69 (1991), p. 141-176.

Duval Noël (s.v.), «Édifices de culte», in Angelo di Berardino (dir.), Dictionnaire encyclopédique du christianisme ancien, Paris, 1990, p. 752-771.

69. Rationale div. Off., I, VI, 5, ibid., p. 66-67. 
Duval Noël, « Problématique d'une architecture chrétienne au IV siècle », Revue des Études Augustiniennes, 35 (1989), p. 308-313.

Duval Yvette, Auprès des saints, corps et âme. L'inhumation «ad sanctos » dans la chrétienté d'Orient et d'Occident du III' au VII siècle, Paris, 1988.

Duval Yvettte et PICARD Jean-Charles (éds.), L'inhumation privilégiée du IV e au VIII s. en Occident. Actes du colloque tenu à Créteil les 16-18 mars 1984, Paris, 1986.

ERMINI PANI Letizia, « Il cristianesimo nell' antichità e nell'alto medioevo », in André Vauchez (dir.), Lieux sacrés, lieux de culte, sanctuaires, p. 107-117.

FÉVRIER Paul-Albert, « Remarques sur le paysage d'une ville à la fin de l'Antiquité. L'exemple d'Aquilée », Antichità Altoadriatiche, 19 (1981), p. 163-212.

FosCHIA Laurence, «La réutilisation des sanctuaires païens par les chrétiens en Grèce continentale (IV ${ }^{\mathrm{e}}-\mathrm{VII}^{\mathrm{e}} \mathrm{s}$.) », Revue des études grecques, 113 (2000), p. 413-434.

GANDOLFo Francesco, «Luoghi dei santi e luoghi dei Demoni », in Santi e demoni nell'alto medioevo occidentale (secoli $V-X I$ ), 36 ${ }^{\mathrm{e}}$ SSCISAM, Spolète, 1989, p. 883-916.

Kraeling Carl H., The Excavations at Dura Europos, Final Report VIII, Part II. The Christian Building, New Haven, 1967.

KAMPLING Rainer, « Die Darstellung der Juden und des Judentums in den Predigten des Zeno von Verona », Kairos, 26 (1984), p. 10-24.

KRAUTHEIMER Richard, Rome, portrait d'une ville, trad. F. Monfrin, Paris, 1999, (Princeton, 1980).

KRAUTHEIMER Richard, Tre capitali cristiane. Topografia e politica, Turin, 1987 (Berkeley-Los Angeles-Londres, 1983).

MARKUS Robert A., "How on earth could places become holy?", Journal of Early Christian Studies, 2 (1994), p. 257-271.

Markus Robert A., The End of Ancient Christianity, Cambridge, 1993.

MoHRman Christine, «Les dénominations de l'église en tant qu'édifice en grec et en latin au cours des premiers siècles chrétiens », in Études sur le latin des chrétiens, IV, Rome, 1977, p. 211-230.

Monfrin Françoise, «À propos de Milan chrétien. Siège épiscopal et topographie chrétienne $\mathrm{IV}^{\mathrm{e}}-\mathrm{VI}^{\mathrm{e}}$ siècles », Cahiers Archéologiques, 39 (1991), p. 7-46.

MoRANI Moreno, «Sull'espressione linguistica dell'idea di santuario nelle civiltà classiche », in Marta Sordi (dir.), Santuari e politica nel mondo antico, Milan, 1983, p. 9.

NeSTORI Aldo, « Riflessioni sul luogo di culto cristiano precostantiniano », Rivista di Archeologia Cristiana, 75, 1-2 (1999), p. 695-709.

PERRIN Michel Yves, «Le nouveau style missionnaire. La conquête de l'espace et du temps », in Jean-Marie Mayeur et al. (dir.), Histoire du 
christianisme. 2. Naissance d'une chrétienté (250-430), Paris, 1995, p. 585-621.

PIETRI Luce, «Grégoire de Tours et la géographie du sacré », in Nancy Gauthier et Henri Galinié (éds.), Grégoire de Tours et l'espace gaulois : actes du congrès international, Tours, 3-5 novembre 1994, Tours, 1997, p. 111-114.

QUACQUARELli Antonio, «I luoghi di culto e il linguaggio simbolico nei primi due secoli cristiani », Rivista di Archeologia Cristiana, 42 (1966 [1968]), p. 237-266.

REBILLARD Éric, «Violations de sépulture et impiété dans l'Antiquité tardive », in L. Mary, Michel Sot (éds.), Impies et païens entre Antiquité et Moyen Âge, Paris, 2002, p. 65-80.

REEKMANS Louis, "L'implantation monumentale chrétienne dans le paysage urbain de Rome de 300 à 850 », in Actes du XI Congrès international d'archéologie chrétienne. Lyon, Vienne, Grenoble, Genève et Aoste (21-28 septembre 1986), Rome-Cité du Vatican, 1989, p. 861-915.

SCHUSTER Ildefonse, Liber Sacramentorum, 8, Turin, 1927.

SMith Jonathan Z., To Take Place: Toward Theory in Ritual, Chicago, 1987.

Sotinel Claire, «La disparition des lieux de culte païens en Occident. Enjeux et méthode », in Michel Narcy et Éric Rebillard (éds.), Hellénisme et christianisme, Villeneuve d'Ascq, 2004, p. 35-60.

SotinEl Claire, « Lieux de culte et sanctuaires dans le christianisme ancien. Enquête bibliographique », in Lieux sacrés, lieux de culte, sanctuaires, op. cit. note 10, p. 95-105.

Sotinel Claire, «Locus orationis ou domus Dei ? Le témoignage de Zénon de Vérone sur l'évolution des églises (tractatus II, 6) », Studia Patristica, 29 (1997), p. 141-147.

Sotinel Claire, Identité civique et christianisme. Aquilée du III au vie siècle, Rome, sous presse.

TAYlor Joan E., Christians and Holy Places: The Myth of Jewish-Christian Origins, Oxford, 1993, p. 308.

Testini Pasquale, Cantino Wataghin Gisela et PAni Ermini Letizia, $\mathrm{La}$ cattedrale in Italia, in Actes du XI ${ }^{e}$ Congrès international d'archéologie, p. 5-87.

VAUCHEZ André (dir.), Lieux sacrés, lieux de culte, sanctuaires. Approches terminologiques, méthodologiques, historiques et monographiques (Collection de l'École française, 273), Rome, 2000.

Walker Peter W. L., Holy City, Holy Places? Christian attitudes to Jerusalem and the holy land in the fourth century, Oxford, 1999.

WARD PERKINS John Bryan, «Constantine and the origin of the Christian basilica », Papers of the Bristish School in Rome, 22 (1954), p. 69-90 = Studies in Roman and Early Christian Architecture, Londres, 1994, p. 363-383. 